INTERNATIONAL JOURNAL OF MULTidisciplinARY RESEARCH AND ANALYSis

ISSN(print): 2643-9840, ISSN(online): 2643-9875

Volume 05 Issue 02 February 2022

DOI: 10.47191/ijmra/v5-i2-09, Impact Factor: 6.072

Page No.- 300-302

\title{
Combinatorial and Giant Combinatorial Scattering
}

\author{
M.D.Nomozova \\ PhD, National University of Uzbekistan named after Mirzo Ulugbek
}

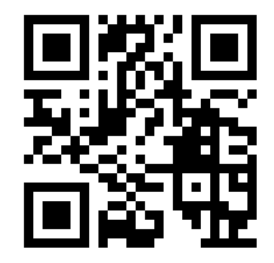

ABSTRACT: This article substantiates the importance of introducing the topic "Raman and giant Raman scattering of light" into the course "Nanophysics", taught to the students of the Faculty of Physics of the National University of Uzbekistan named after Mirzo Ulugbek. The essence of Raman scattering of light is explained from the point of view of quantum mechanics. Detailed information is given on the polarization, the dipole moment of molecules, and on the stokes and anti-stokes components of the spectrum of Raman scattering of light. The reason for the low intensity of the anti-stokes component of Raman scattering of light is given. New knowledge related to giant combinatorial scattering in nanomaterials will also be discussed.

KEYWORDS: Substantiates, Importance, Raman, Light, Scattering, Anti- Stokes Component, Quantum Mechanics.

\section{INTRODUCTION}

It is known that the development of modern technologies is based on the latest achievements of science and science. We all know that without new knowledge, new technologies will not emerge. That is why students studying in higher education institutions must constantly acquire new knowledge. That is why the course of "nanophysics" has been taught at the Faculty of Physics of the National University of Uzbekistan named after Mirzo Ulugbek for 3 years. With this in mind, it is a good idea to include the topic "Combinatorial and giant combinatorial scattering" in the curriculum of "nanophysics". Well-versed in the nature of the phenomena of combinatorial and giant combinatorial scattering of light, future specialists will have no difficulty in applying the combined spectroscopy of nanomaterials in many fields, such as pharmaceuticals, medicine, biomedicine. The phenomenon of combinatorial scattering can be explained on the basis of classical wave theory of light as well as quantum physics. From the point of view of classical physics [1,2], light is considered to be electromagnetic waves, and molecules are polarized as a result of the interaction of these waves with the environment.

\section{THE MAIN FINDINGS AND RESULTS}

Polarization depends on the interaction of the "electron cloud" of atoms with the electric field of electromagnetic waves. In quantum theory, light is thought to be a stream of photons, and scattering occurs when they collide with molecules. The number of scattered photons depends on the strength of the bonds in the molecules. The classical theory of ordinary combinatorial scattering is studied in the optics department of general physics. Before studying the essence of giant combinatorial scattering, we found it necessary to briefly dwell on the quantum theory of combinatorial (Raman) scattering $[3,4]$.

\section{QUANTUM THEORY OF RAMAN SCATTERING}

The combination scattering or Raman effect is the inelastic scattering of photons from environment molecules. As shown in the diagram in Figure 1 [4], the light photon awakens the molecule and transfers it to a virtual state. Here, three cases are observed. First, a molecule in the state of the basic zero oscillation level absorbs the falling light photon, emits a new photon equal to its energy, and returns to the ground state. This process represents the relay effect. 


\section{Combinatorial and Giant Combinatorial Scattering}

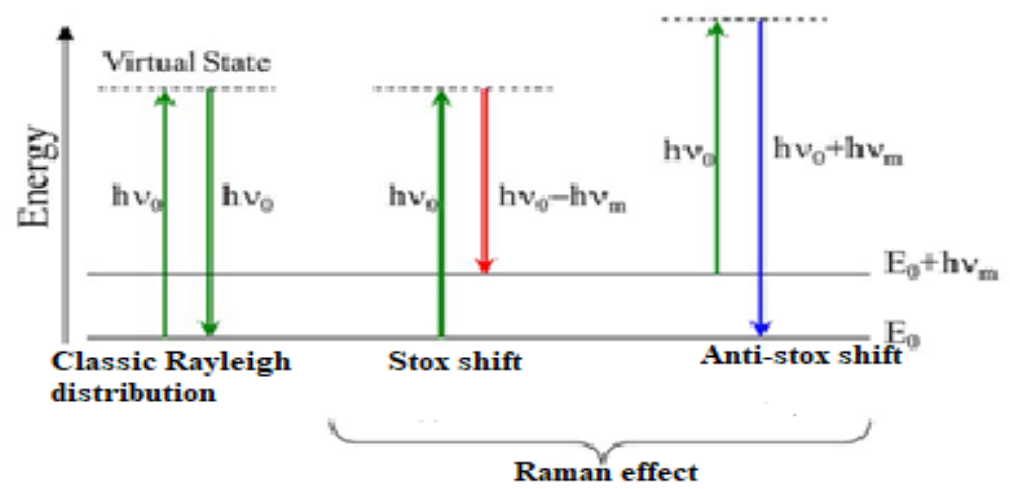

Figure 1. Explain stokes and Antistokes shifts in terms of quantum theory

The second is that the molecule swallows the falling light photon, passes from the ground state to the virtual state, and descends to the first awakened level of the molecule's vibration. In this case, the molecule emits part of the absorbed photon energy as a photon. This process is called Raman scattering. In the third case, the molecule passes from the first awakened state of the phonon to the virtual state and emits a photon with a higher energy than the incident light photon. This effect is referred to as the antistokes shift of Raman scattering. The intensity of the antistokes component of the combination spray is very small. To understand the cause of this condition, let us recall the Boltsmann distribution. It is known that the employment of vibration surfaces under conditions of heat equilibrium is subject to the Boltsmann distribution. According to this distribution, the employment of the surfaces decreases according to the exponent law. At room temperature, the probability of scattering is low because most of the molecules are in the ground state and the occupancy rate in the first awakening state of the phonon is very low. Therefore, the intensity of the anti-stokes spectral lines in the combinatorial scattering (CS) spectrum is much lower than in the stock lines. Typically, the CS spectrum refers to the stock portion of the highintensity spectrum. As a result, Raman scattering measurements are performed during the stock stock displacement process.

Raman scattering cross section $\sigma \mathrm{R}$ is determined as follows:

$$
\sigma_{R} \propto \frac{1}{\lambda^{4}}
$$

Here, $\lambda$ - the wavelength of the falling photons

The power of scattered light $P_{s}$ and the intensity of the incident light lo linked by the following formula:

$$
P_{S} \propto \frac{I_{O}}{\lambda^{4}}
$$

(2) - according to the formula, the intensity of the scattered light is directly proportional to the intensity of the incident light and inversely proportional to the 4th degree of wavelength. Therefore, it is advisable to use an alarm source with high intensity and short wavelength to increase the power of scattered light.

\section{GIANT COMBINATORIAL SCATTERING}

The giant increase in combinatorial scattering was first observed in 1974 by M. Fleishman [5]. M. Fleishmann experimentally observed a combination scattering of molecules deposited on an uneven (rough) silver surface. The main goal of the experiment was to increase the number of molecules deposited on the silver surface by increasing its surface area. However, the scattering intensity of the molecules was much greater than expected. In many foreign countries, this effect is known as "Surfaceenhanced Raman scattering" (SERS), In 1978, it was found that the proportion of Raman photons in the scattered light of molecules adsorbed on the rough surfaces of metals increased $10^{6}-10^{7}$ times. This phenomenon is called "giant combinatorial scattering of light". In 1997, exotic nanoobjects (their share was $10^{-4}$ ) were found in colloidal solutions of silver nanoparticles (NS). It was found that the number of Raman photons in these nanoobjects increased $10^{14}$ times. Subsequent investigations revealed that these nanoobjects were a pair of interconnected nanoparticles called dimers. It was also found that the reason for such a fantastic increase in the number of Raman photons was due to the appearance of dimers. In the narrow gap between the dimer nanoparticles, the adsorbed molecule falls into a very large electric field of a specific "nanocondenser". Because the Raman effect is proportional to the fourth level of the electric field, the area of the crack is thousands of times larger than the electric field of the incident light wave. As a result of this increase in the effect, it is possible to measure the Raman spectrum of a particular molecule adsorbed on the dimer. 


\section{Combinatorial and Giant Combinatorial Scattering}

\section{CONCLUSION}

Nanoparticles can be thought of as active nano antennas that perform the following functions: receiving an electromagnetic signal; conversion of the signal into surface plasmons; amplification of the signal in the form of a near field in the slit where the dipole is located; receiving the Raman signal of the dipole shifting in frequency, amplifying the resonance, and redistributing this radiation into space. As we know (the topic of "surface plasmon resonance in metal particles"), under the influence of the oscillating external field, the free "electron gas" oscillates and extends exponentially inside the nanoparticle. This phenomenon is surface plasmons. The plasmon resonance of gold, silver, and metallic nanoparticles occur in the visible range of light. The wavelength of surface plasmons is much smaller than the wavelength of light. Therefore, it is possible to compress the electric field inside the slit using plasmas. In this way it is possible to get rid of the ban imposed by physical optics on the size of the focal spot of light.

\section{REFERENCES}

1) Landsberg G.S. Optics, ed. "Science", Moscow: 1976.

2) Landsberg G.S. Optics Teacher Publishing House, Tashkent:1981

3) Pentin Yu.A., Kuramshina G.M. Fundamentals of Molecular Spectroscopy. Moscow: World, 2008.

4) Kovalenko A.A., Eliseev A.A., Methodical development of Raman spectroscopy, Lomonosov Moscow State University M.V. Lomonosov,2011.

5) Fleisshmann M., Gendra P. J., McQuillan A. J. Raman Spectra of Pyridine Adsorbed At a Silver Electrode, Chem. Lett. 1974. Volume 26, № 2. 